\title{
Impression Evaluation and Management of Teachers of University
}

\author{
Chengfang Liu ${ }^{1}$ Wei Liu ${ }^{2}$ \\ ${ }^{1}$ Office of elites' introduction, Southwest university for nationalities, Chengdu,Sichuan, 610041,China \\ 2.College of economics, Southwest University for nationalities, Chengdu, Sichuan, 610041, China
}

\begin{abstract}
As we known, teachers play an important role in training qualified personnel and researching in university. This paper discusses the problem of impression evaluation and management of teachers of university. An index system of evaluation of the impression management is presented. We put forward eight aspects of applied strategies of impression management.
\end{abstract}

Keywords: Teachers of university, Impression evaluation, Impression management

\section{Introduction}

The impression evaluation is the process that an individual influences others to form an impression on himself. Influenced by motive of improving oneself and multiple ego performance, the impression management of oneself is divided into acquisitive impression management and protective impression management. The multiple roles of teachers of university come to a decision that they should be able to have a good command of interpersonal relationships. In the process of educating and teaching activities, on the basis of right moral values, felicitous application of strategies of the impression management will help teachers to enhance the interpersonal communication and be more harmonious, smooth and successful.

The theories of impression management supposed that an individual exists a kind of basic motive, namely in spite of the individual being a member of the organization or not, who hope the other people treat actively, and avoid being been negative by the other people to treat (Rosenfeld, 1997). The impression management of individual influenced by the motive of ego build-up and ego performance .Ego build-up is the basic motive of human beings, in the social communications, people always try their best to manifest their excellence, eager to be regarded actively, shadow their shortcomings to avoid being considered negatively. An individual has a trend to show himself. Willian James (Leary \& Kowalski, 1990) thought in his work is the psychology principle. It denoted that people had multiple egos but not the simple ego concept only, and people can express dissimilarity in the different situation. That is to say, for the sake of pleasing in the different objects, people would appear multiple society egos skillfully.

Arkin (1981) divided the concept of impression management into acquisitive impression management and the protective impression management. The former one is regarded as the function of subjective possibility for performing oneself that stimulate value and realize success, for making others recognize one's great efforts positively to win the identification and acclimation; the later one aims to avoid the obvious loss of social identification and the accumulation of social disrecognization, weaken one's shortcomings and avoid being considered negatively to take protective measures as possible as one can, to renovate the weakened image .In his eyes. The main value of individual's impression management lies in being as an effective and reliable index to evaluate individual's qualification. Taking a risk is the main performance of acquisitive impression management; protection impression management expressed being less participation in the society interaction, few words at the beginning, also say not much afterwards and get around may announce to public stupid of the topic, the minimal ego expose and describe modestly and by one's own. With this mutually consistent motive modes are defensive impression management and the affirmation impression management. The basic differentiation is that the former is the social image that designs to protect individual to maintain built- up image, and it is led by the negative emotion appearance to move, being a consciousness to of to oneself the threat of the social image activate; the latter is the social image that designs and improves individual image, it is strengthened by one's motive of improving oneself, stirred up by the opportunity that be consciousness of possible pleasant the impression.

What People perform according to do? Goffman (1959) thought that people perform with the purpose of setting up a hopeful the impressions in the others heart. 
This paper aims at improving the level of impression of teachers of university. The main content of this paper includes four aspects, and they are the concept of impression management, a model of evaluation of teachers of university, Strategies of impression management for teachers of university and conclusion respectively.

\section{The Concept of Impression Management}

People usually make use of the interaction of information to attain the aim of interpersonal association. The impression management means the process that an individual tries to control others' impression on him with languages and behaviors to form a certain expectant impression, which is also called impression grooming. Since 1950's, the research of the impression management has got a quick development in the sociology, psychology, the management learn etc.

American famous sociologist Goffman (1959) started the research of the impression management. in his opinion, the interests of one of the mutual aspects lies in controlling others' behaviors, let others understand their behaviors, make them response properly in line with one's previous expectation. His thought-developed ways for a series researches that social psychology manages concerning the ego and impression afterwards.

Then, Jones (1982) and the alike extended the ego performance to the containment scheme to control others owning personal impression of characteristics, which raised the great interest of psychologists on the impression management, changed the situation that the impression management only belongs to the sociology category.

In the 1980s, experts carried on re-analyzing to the concept of the impression management. From the point of the scene definition, Schlenker (1985) put forward expect-value model, he thought that the impression management meaned purposely or unpurposely trying to control the impression on the interaction of the real or imaginative society. Arkin (1981) also began from the scenario definition, thought that as a kind of process; the impression management meant that individual planed, adopted, carried out the delivery of the ego image with others in the interactive scenario.

Leary and Kowalski (1990) thought that the impression management included two different compositions or processes. (1) The individual tries the control others to own impression wishes or the motive, which is called impression motive. (2) The individual decides what kind of impression on others' minds, and how this kind of impression formed, which id called impression construction.

Rosenfeld (1997) thought that the impression management was the process that a kind of people tried the control others to become a certain impression to the oneself.

According to the literatures presented, the impression management means the impression process that the individual tries the control others to form the impression of oneself.

Others' impression on him showed that others' felling, evaluation even the specified ways to someone. An individual always hopes to keep a fine impression for others; it is indispensable to have the valid and fitting impression management to keep fine interpersonal relationships. The impression management is the way that an individual apt to social life to realize the social expectation.

\section{A model of evaluation of teachers of university}

According to the content of impression management, we build a model of evaluation of teacher of university that is shown as Fig.1.

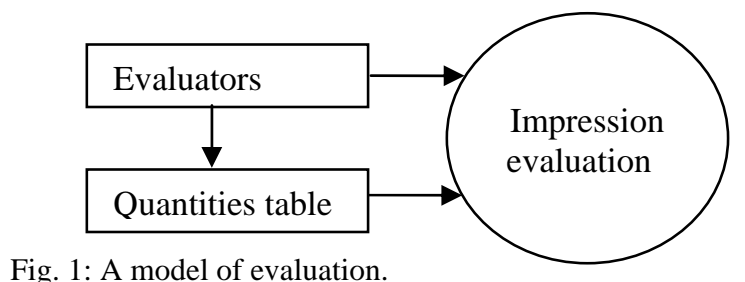

In Fig.1, because there are many evaluators, such as expert, director of HR and leader of the department etc., the power weight should be considered in the process of evaluation. The quantities table is the system of index of evaluation actually.

We put forward eight aspects of index of evaluation, i.e. example impression, first impression, appetency impression, publicize ego impression, magic power impression, friendly impression, authority impression, humble impression.

The model of evaluation of impression of teacher of university can be expressed by the following formula.

$$
R I=\sum_{i=1}^{n} w_{i} \times(A \circ R)
$$

In formula (1), the symbol of $R I, w_{i}, A$ and $R$ denotes the final result of evaluation, the $\mathrm{i}$-th evaluator's power weight, the vector sets of power weight of quantities table and the result of quantities table respectively.

Due to fuzzy information existing in the course of 
evaluation, the method of fuzzy comprehension evaluation is suitable to the evaluation of impression.

\section{Strategies of impression management for teachers of university}

Teachers of universities play multiple roles in their work.. From the point of the task of profession, as the professionals, teachers of university are not only in charged with the work of imparting knowledge and educating people, but also take on the tasks of scientific research of discover and create knowledge. From the course of working, their jobs are represented with more obvious technicality, go-aheadism, independence and creativity. From the roles of their playing, they impart knowledge and educate people as well as doing academic research, performing the tasks of education as well as managing the activities of educating and teaching. Besides, they have to be supervised and evaluated by leaders of all levels, experts and students. The multiple roles of teacher of university demand that they have extensive interpersonal communications. This paper researches how to improve the effectiveness of teachers of university properly by using the strategic of impression management in the process of educating and teaching activities.

How let the students, colleagues and leadership approve someone? According to the characteristics of profession and roles of tutors, on the basis of the right morals evaluate, from acquiring sex impression management and the protection impression management to set out, the author put forward the impression management strategies for tutors of universities and colleges as following.

A. Being example for others, to set up a example impression

The life in universities and colleges is exactly the important stage of a students' global view, value and the health personality formation. A tutor is the model for a student in the course of imparting knowledge and educating people , the enterprise, responsibility, academic ideal, attitude of pursue of one's studies will influence students' inner mind which functions both at students' learning time at universities and all the life after graduation and affects the overall quality of human beings in real society and future. Because it is now in the stage of popularity of higher education, the learn to teach, behave to guide, tutors of universities and colleges should enhance self -culture morally and academically, make oneself the model for students learning all the life. This is the core that the tutor's impression manages in the relationship of tutors and pupils.
B. Controlling the first timing, to enhance the first impression

In the interpersonal communications, the impression left at the beginning usually is strong and deep, this kind of phenomenon on the psychology is called "first reason effect". Students first impression on teachers will influence the teaching work directly and hardly to change. While contacting student's first time the teacher wants to control active power of good prejudiced association, value appearance, talk-to-talk bearing and ability and learning wise exertive, work hard to create the best first impression, lay good foundation for the follow-up education.

C. Sincerely laud, to set up appetency impression

The social psychology research expressed that people were inclined toward fancy to like an oneself or evaluate actively to the oneself of person. Admiring others sincerely will please oneself, students, colleagues and administers. Successful praise demands the following three points: Firstly, bear firmly in mind the most true sayings of there must be a teacher among three people, keep the modest attitude then can discover the others' advantages, then can promise the praise hair from the heart. Secondly, be good at passing the third square to present the one's compliment to the other people. The third, strengthen the credibility of the phrase of praise, teacher while praising students, colleagues or leadership should fully consider the situation, opportune moment, frequency and distinction.

D. Displaying appropriately, to publicize ego impression

The fitting ego demonstration is the important strategy of the impression management. People trend to favor those who consider himself is capable. Display the own strength, the personality magic power and the research result in the student's in front by suitable form, carry on blowing one's own trumpet actively, contribute to the good image of the further establishment teacher. self- revelation should follow the principle of gradualism that properly control making the two sides comfortable in the course of revelation. A point all not display or display too many may cause the negative reaction of the other party too quickly, disadvantage in the establishment of the good impression.

E. Establishing a scenario, to mold a magic power impression

The researches of psychology express that the activity that takes to have strong motion to experience personally can bring into the person's long time memory system unconsciously, leaving a deep impression for person. Therefore, tutors should have a good command of creating emotional environment, integrating people with environment, sense and 
sensibility, awareness and acts and so on organically, giving powerful convulsion to others and form characteristic individual charming. Moreover, the facial expression, the physique language and human relations and alike the non- language behavior can influence of the teacher and others availably an impression mutually. Teachers of university should pay attention to their behaviors of non-language such as bearing, clothes, decoration that should be submitted with environment to avoid the unpleasant impression of being against the grain.

F. Obeying reasonably, to build a friendly impression

Because of others' participation in some one activity, someone also is engaged in this activity or gives up an own opinion but adopts the opinion of the others; this kind of phenomenon is called to follow. In dealing with some non-principle problems, tutors should take reasonable strategies to avoid supercilious and haughty impression for high degrees or high titles of a technical post. The equilibrium mode of the social psychology point out that people are all inclined towards the choice consistency, then people always like those and one's opinion the consistent person is together. There are two strategies of reasonable obey: the one is that express one's point of view to enhance one's reliability when there are some disagreement among the accordance; the other is that express one's different opinions firstly and then turn to agree gradually on the basis of unceasing give-way, which can raise one's popularity and build up imagine of independence.

G. Using distance, to set up authority impression

Such as above said, Goffman made the interaction kept the certain distance square, making the other party produce a kind of impression management that respects adore mental state to call it as "the mystification perform" the social psychology research indicates that despite that acquaintance can raise likeness, the more the acquaintance, the much more feeling of belittle will bring. The purpose that the role insulates is the behavior that avoid both parties canning see the other party "backstage" mutually. The proper subtle distance between tutors and objects will do good for constructing authorized imagine.

$\mathrm{H}$. Apologizing in time, to keep humble impression

To some degree, the attitude of apologizing means to admit responsibility of oneself, and own behavior self-reproach and regretfully, acquire the pardon of the others by period. Apologizing is a main way of protective impression management strategies. Wise of the usage apologize, admitting an own mistake; go toward can acquire the good will of the student or governor on the contrary and more. Apologizing requires clear statement, regretful expression, and strict self-scritism and asks for pardon and put forward rectifying or repairing suggestions.

\section{Conclusions}

The paper took the impression as an aggressive concept. On the basis of right moral evaluation, an individual tries to influence, control others' minds and acts on him and avoid imagine being weakened, which is the representation that individual highly apt to the society. Reasonable impression management not only is inevitable, but also is expect in the human relations the association of the culture and the social phenomena. It's obvious that there is many factors affect the result of evaluation of impression of teacher of university. To improve the level of university management, we should pay more attention to the impression evaluation and management of teachers.

\section{Acknowledgment}

We gratefully acknowledge the support of National Social Science Foundation of Sichuan (SC06A013).

\section{References}

[1] J. T. Tedeschi editor, Impression management theory and social psychological research, $R$. M. Arkin, Self-presentation Style, New York, Academic Press, pp.311-333, 1981.

[2] E. E Coffman, The Presentation of self in Everyday life New York, Doubleday Ancher, 1959.

[3] C. A. Higgins, T. A. Judge and G. R. Ferris, Influence tactics and work outcomes: A metaanalysis. Journal of Organizational Behavior, 24: 89-106,2003.

[4] K. M. Kacmar, and D. S. Carlson, Effectiveness of impression management tactics across human resource situations, Journal of Applied Social Psychology, 29:1293-1315,1999.

[5] M. R. Leary, and R. M. Kowalski, Impression management: A literature review and two components model, Psychological Bulletin, 1990.

[6] L. A. McFarl A M Ryan, and S. D. Kriska, Impression management use and effectiveness across assessment methods, Journal of Management, 29(5): 641-661,2003.

[7] P. Rosenfeld, Impression Management, Fairness, and the Employment Interview, Journal of Business Ethics, 16(8): 801-808,1997.

[8] B. R. Schlenker, Editor, The self-and social life: Identity and self-identification, New York, McGraw-Hill, pp. 65-99,1985. 